\title{
SERUM TOTAL AND BONE ALKALINE PHOSPHATASE LEVELS AND THEIR CORRELATION WITH SERUM MINERALS OVER THE LIFESPAN OF SHEEP
}

\author{
Cristina P. SousA ${ }^{1,2,3}$, Jorge T. De Azevedo ${ }^{4,5}$, Amélia M. Silva ${ }^{6,7}$, \\ Carlos A. VIEGAS ${ }^{1,2,3}$, Rui L. ReIS ${ }^{2,3}$, Manuela E. Gomes ${ }^{2,3}$ and Isabel R. DIAS ${ }^{1,2,3^{*}}$ \\ ${ }^{1}$ Department of Veterinary Sciences, ECAV - University of Trás-os-Montes e Alto \\ Douro (UTAD), P.O. Box 1013, 5001-801 Vila Real, Portugal; ${ }^{2} 3 \mathrm{~B}$ 's Research Group, \\ Department of Polymer Engineering, University of Minho, Guimarães, Portugal; \\ ${ }^{3}$ ICVS/3B's - Life and Health Sciences Research Institute, Braga/Guimarães, Portugal; \\ ${ }^{4}$ Department of Animal Sciences, ECAV-UTAD; ${ }^{5}$ CECAV - Center for Animal Sciences \\ and Veterinary Studies, UTAD, Vila Real, Portugal; ${ }^{6}$ Department of Biology and \\ Environment, ECVA-UTAD; ${ }^{7} \mathrm{CITAB}$ - Centre for Research and Technology of \\ Agro-Environmental and Biological Sciences, UTAD, Vila Real, Portugal
}

(Received 10 December 2012; accepted 2 April 2013)

This study aimed to assess serum total alkaline phosphatase (ALP) and its bone isoform (BALP) levels during the ageing and in different physiologic states of sheep, in order to expand the knowledge about the variation of these biomarkers over the sheep lifespan. Ninety female sheep were divided into nine groups of various ages and physiological states (dry, lactation and pregnancy). Serum ALP, BALP and mineral levels were determined by commercial immunoassay, molecular absorbance spectrophotometry and chemical luminescence for BALP determination. Serum ALP and BALP decreased as sheep aged, and no statistically significant differences were obtained between ewes in different physiologic states. The continuous decline of serum BALP concentration along the sheep lifespan, namely in mature and old sheep, is a sign of decreasing bone turnover associated with ageing. Serum calcium concentrations increased slightly until 2 years of age and then showed a tenuous but statistically significant decrease in mature sheep, while serum phosphorus maintained an uninterrupted decrease as sheep matured. The knowledge of serum values of bone biomarkers throughout the sheep lifespan may be useful in preclinical orthopaedic research studies and for animal science studies using sheep.

Key words: Total alkaline phosphatase, bone alkaline phosphatase, minerals, sheep, ageing, physiologic state

Bone biomarkers are subdivided into formation and resorption markers. The bone formation markers are the products of osteoblastic activity formed dur-

*Corresponding author; E-mail: idias@utad.pt; Phone: 00351 (259) 350-632;

Fax: 00351 (259) 350-480 
ing the different stages of osteoblastic proliferation and differentiation - bone alkaline phosphatase (BALP) and osteocalcin (OC), and osteoid synthesis - the amino-terminal (PINP) and carboxy-terminal propeptides of collagen type I (PICP) (Seibel, 2006). The markers of bone resorption include the products of type I collagen degradation - the N-terminal (NTX) and C-terminal telopeptide (CTX) and ICTP (a slight variant of CTX), pyridinolines - the end-products of the NTX and CTX metabolism, hydroxyproline and hydroxylysine, and the proteolytic enzymes secreted by the osteoclasts - tartrate-resistant acid phosphatase (TRAP) (Seibel, 2006). These biomarkers could provide a simple and sensitive method for supporting the diagnosis of high-turnover bone disorders that focally involve the skeleton or diffuse disorders, where their levels usually increase in serum and urine, and have been recently used in human medicine for the assessment of post-menopausal osteoporosis and Paget's disease and to monitor their treatment (Seibel, 2006).

In veterinary medicine, namely in sheep, bone biomarkers have also been used, namely total alkaline phosphatase (ALP), BALP, OC, PICP, pyridinolines (Allen, 2003) and also TRAP (Seebeck et al., 2005), for animal science studies and as a complementary method of experimental orthopaedic research using sheep, namely to evaluate the fracture healing process (Klein et al., 2004; Seebeck et al., 2005), the pathophysiology of post-menopausal osteoporosis (Sigrist et al., 2007; Turner, 2007) and for the development of new implants for fracture treatment in osteoporotic bone (Egermann et al., 2008). Preclinical studies aimed at assessing fracture healing processes and/or related complications usually use skeletally adult ( 2 to 6 years old) animals, with no interference of the increasing osteogenic potential present during growth. For preclinical studies of post-menopausal osteoporosis, usually 8 to 10 years old ewes are used after ovariectomy (Turner et al., 1995) or after a corticoid administration protocol (Chavassieux et al., 1997). Also, adult sheep subjected to an induction regimen consisting of ovariectomy, to promote oestrogen deficiency, associated with a specific calcium-/vitamin Drestricted diet and/or a glucocorticoid medication protocol have been used (MacLeay et al., 2004; Ding et al., 2010). Furthermore, in animal science studies on sheep, bone markers have already been used for monitoring alterations in bone turnover during growth or due to different physiological demands during the female reproductive cycle, where high calcium requirements occur and mineral homeostasis and bone metabolism could overcome significant changes (Allen, 2003; Liesegang et al., 2006), or even to evaluate the diurnal and circadian variations of these biomarkers (Liesegang et al., 2003).

One of the most widely used biomarker of bone formation is a membranebound enzyme (ALP), its activity being the sum of four isoenzymes in humans: the intestinal ALP (IALP), the placental ALP, the germ cell ALP, and a tissue non-specific ALP (TNSALP) present in the liver, kidney and bone (Millán, 2006), each of them a product of different gene loci (Goldstein et al., 1980). Al- 
though the TNSALP derives from the same gene locus, BALP and the liver isoform differ in an organ-specific post-translational glycosylation, which allows their measurement by specific analytical biochemical immunoassays. These two isoforms represent the most relevant fraction of the total ALP activity, with an almost equal contribution to about $95 \%$ of this enzyme (Millán, 2006). Sheep present only the IALP and the TNSALP (Goldstein et al., 1980).

BALP is one of the most sensitive markers of bone formation. It is synthesised and expressed at relatively high levels by osteoblasts simultaneously with the secretion of osteoid (Seibel, 2006). BALP activates the hydrolysis of phosphate esters on the osteoblast cell surface supplying a high extracellular inorganic phosphate (iP) concentration for osteoid mineralisation and also promoting the breakdown of pyrophosphates, which are inhibitors of ionised calcium and iP deposition into osteoid (Whyte, 1994; Millán, 2006).

Since sheep have been used in animal science studies and also as a large animal for orthopaedic research involving measurements of bone metabolism biomarkers, namely serum ALP and BALP levels, the aim of this study was to measure the values of these biomarkers and to evaluate the correlation between them and the serum minerals in sheep of various ages and different physiologic stages, in order to expand the knowledge about the variation of these biomarkers occurring over the lifespan of sheep.

\section{Materials and methods}

\section{Animals and housing}

This study was approved by the National Animal Use and Care Committee and undertaken according to the regulations of the European Community Legislation on Animal Care. Ninety healthy Assaf female sheep were randomly chosen from a sheep flock and grouped according to age and/or physiologic state (10 animals/group): 1 month old and 6 months old lambs; non-lactating and nonpregnant 1 year old, 2 years old, 3 to 5 years old, 6 to 8 years old sheep and elderly ewes over 8 years of age; 3 to 5 years old ewes with 2 to 3 months of lactation or in the middle of pregnancy. The flock was kept in a grazing pasture during the day and housed during the night. Diet was based on grass, supplemented with $0.250 \mathrm{~kg} /$ day of concentrate/animal, and water was provided ad libitum. The diet provided approximately 1.20 times the energy maintenance requirements according to the National Research Council recommendations (NRC, 1985) (Table 1). Samples of hay and concentrate were taken weekly and dried $\left(65^{\circ} \mathrm{C}, 24 \mathrm{~h}\right)$ to determine dry matter content. Dried samples of feeds were ground to pass a 1-mm screen and ash, crude protein and neutral detergent fibre were analysed according to Association of Official Analytical Chemists (AOAC, 1990). 
Table 1

Dry matter (DM) and chemical composition of feeds

\begin{tabular}{lcc}
\hline & Grass hay & Concentrate \\
\hline $\mathrm{DM}(\mathrm{g} / \mathrm{kg})$ & 88.5 & 90.4 \\
Chemical composition $^{*}(\mathrm{~g} / \mathrm{kg} \mathrm{DM})$ & & \\
Ash & 5.9 & 8.5 \\
NDF & 73.3 & 31.6 \\
$\mathrm{CP}$ & 6.1 & 20.7 \\
\hline
\end{tabular}

${ }^{*} \mathrm{CP}$ - crude protein; NDF - neutral detergent fibre

\section{Collection of blood samples}

Jugular blood samples were collected between 9:00 and 10:00 a.m. into serological tubes (S-Monovette ${ }^{\circledR}$, SARSTEDT, Nümbrecht, Germany), centrifuged at $3000 \mathrm{rpm} / 10 \mathrm{~min}$, the resulting serum was harvested rapidly into $2 \mathrm{~mL}$ Eppendorf tubes and stored at $-20^{\circ} \mathrm{C}$ until analyses.

\section{Serum biochemical analysis}

Kits from Abbott Diagnostics (Abbott Park, IL, USA) were used for the measurement of serum ALP activity and serum minerals - calcium $(\mathrm{Ca})$, phosphorus $(\mathrm{P})$ and magnesium $(\mathrm{Mg})$, using a colorimetric method, and read with an automated biochemistry analyser (Aeroset System ${ }^{\circledR}$, Abbott Diagnostics). The measurement of the serum BALP concentration was performed with a commercial immunoassay kit (Ostase ${ }^{\circledR}$, Beckman Coulter, Fullerton, CA, USA) using an automated biochemistry analyser (Access Immunoassay System ${ }^{\circledR}$, Beckman Instruments Inc.) based on chemical luminescence principles, assayed as previously described (Prickett et al., 2008). All samples were measured in duplicate and the mean values were calculated.

\section{Statistical analysis}

Kolmogorov-Smirnov test was used for testing data for normality. The ALP and BALP levels of the experimental groups were compared using a MannWhitney U test. Serum minerals multiple comparison analysis was conducted using ANOVA and a Tukey multiple comparisons range test to compare pairs of means among groups under study. Degree of correlation between the different biochemical parameters and in the different study groups was assessed using a Fisher test. A software programme was used (JMP ${ }^{\circledR}$ 9.0.1., SAS Institute, Inc., Cary, NC, USA) and $\mathrm{P}<0.05$ was regarded as significant difference. 


\section{Results}

Serum ALP and BALP levels did not present a normal distribution but serum minerals did. Serum ALP activities and BALP showed a similar pattern of variation among the age groups with a progressive decrease over lifetime (Fig. 1). Significant differences were found for these parameters between various age groups and their serum levels also showed a wide range of variation over sheep lifetime, with considerable divergence in 1 month old lambs (Fig. 1). Concerning $\mathrm{Ca}$ a slight increase was observed until the age of 2 years, followed by a significant decrease after that age, namely in 3 to 5 years old and over 8 years old sheep (Fig. 2). Serum $P$ revealed an almost continuous decrease as age advanced, with statistically significant difference between 1 month old lambs and 2 years old sheep relative to over 8 years old ewes (Fig. 2). Additionally, 1 month old lambs presented $\mathrm{P}$ differences relative to all other groups. The serum concentration of $\mathrm{Mg}$ was almost identical over the lifetime of sheep (Fig. 2).
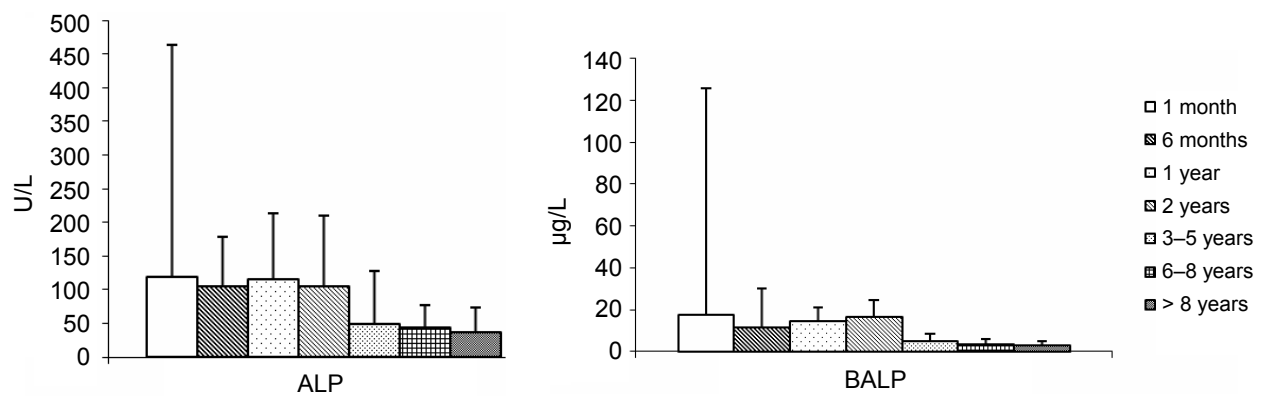

Fig. 1. Serum ALP activity and BALP concentration (median and interquartile range) along the sheep lifespan. Serum ALP activity presented significant differences between 1 month vs. 3-5 years, 6-8 years and $>8$ years $(\mathrm{P}<0.01)$, between 6 months vs. $6-8$ years $(\mathrm{P}<0.01)$, between 1 year vs. $3-5$ years, $6-8$ years $(\mathrm{P}<0.01)$ and $>8$ years $(\mathrm{P}<0.001)$, and between 2 years vs. $3-5$ years, $6-8$ years and $>8$ years $(\mathrm{P}<0.05)$. Serum BALP concentration also presented significant differences between 1 month and 6 months vs. $3-5$ years $(\mathrm{P}<0.01), 6-8$ years and $>8$ years $(\mathrm{P}<0.001)$, between 1 year vs. $3-5$ years, $6-8$ years and $>8$ years $(\mathrm{P}<0.001)$, between 2 years vs. $3-5$ years, $6-8$ years $(\mathrm{P}<0.01)$ and $>8$ years $(\mathrm{P}<0.05)$, and between $3-5$ years vs. $>8$ years $(\mathrm{P}<0.05)$

Concerning the different physiological states of 3 to 5 years old ewes, there was no statistically significant difference for any of the parameters (Figs 3 and 4). Although serum ALP presented an elevated activity in lactating ewes relative to dry and pregnant ewes, the differences were not significant (Fig. 3). In contrast, serum BALP concentration was higher in pregnant ewes than in dry or lactating ones (Fig. 3). Serum Mg concentration was slightly lower in lactating ewes than in dry or pregnant ones (Fig. 4). 


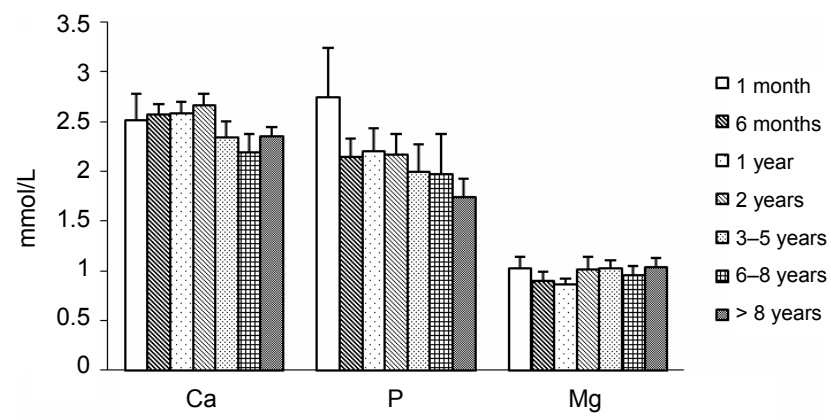

Fig. 2. Serum mineral concentrations (mean $\pm \mathrm{SD}$ ) along the sheep lifespan. Serum Ca level presented significant differences between 1 month vs. $6-8$ years $(\mathrm{P}<0.01)$, between 6 months and 1 year vs. 6-8 years $(\mathrm{P}<0.001)$, and between 2 years vs. $3-5$ years, $6-8$ years $(\mathrm{P}<0.01)$ and $>8$ years $(\mathrm{P}<0.05)$. Serum $\mathrm{P}$ level also presented significant differences between 1 month vs. 6 months $(\mathrm{P}<0.01), 1$ year, 2 years $(\mathrm{P}<0.05), 3-5$ years, $6-8$ years and $>8$ years $(\mathrm{P}<0.001)$, between 6 months and 1 year vs. $>8$ years $(\mathrm{P}<0.001)$, and between 2 years vs. $>8$ years $(\mathrm{P}<0.001)$. Serum $\mathrm{Mg}$ level presented significant difference only between 1 year vs. 2 years $(\mathrm{P}<0.05)$, $3-5$ years and $>8$ years $(\mathrm{P}<0.01)$
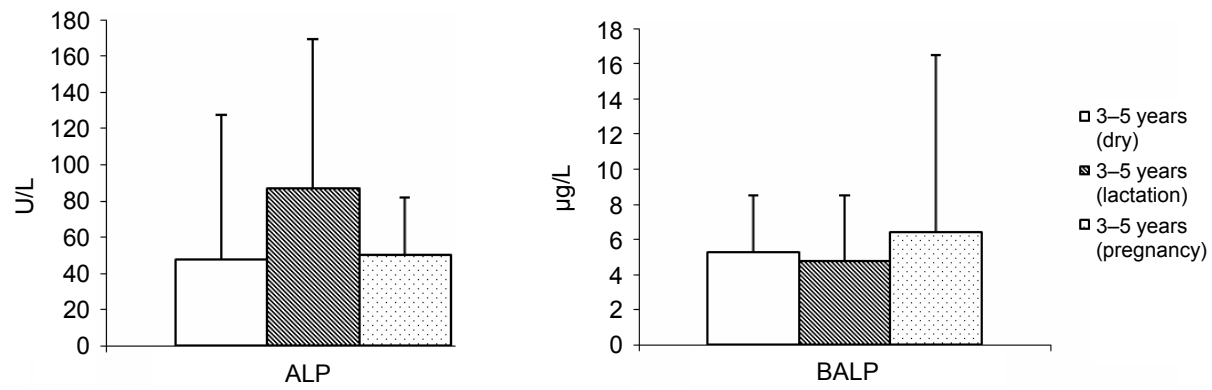

Fig. 3. Serum ALP activity and BALP concentration (median and interquartile range) of 3- to 5year-old sheep of different physiologic states. There was no significant difference between the groups

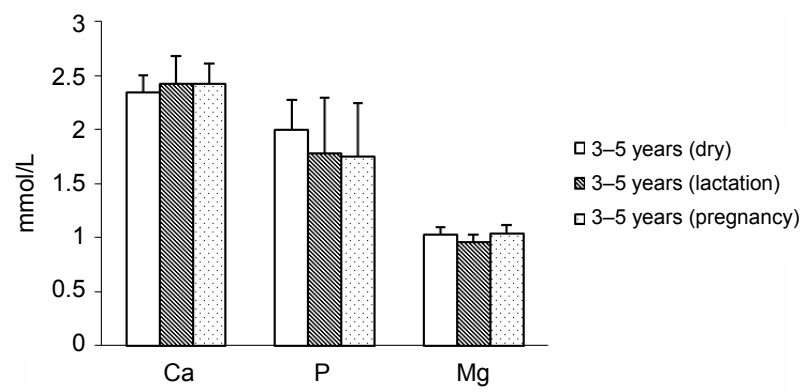

Fig. 4. Serum mineral concentrations (mean $\pm \mathrm{SD}$ ) in 3- to 5-year-old sheep with different physiologic states. The only significant difference was observed in $\mathrm{Mg}$ concentration between lactating and pregnant sheep $(\mathrm{P}<0.05)$ 
Serum ALP activity showed a significant $(\mathrm{P}<0.0001)$ strong correlation with BALP $(\mathrm{r}=0.96)$, and a medium correlation with $\mathrm{Ca}(\mathrm{r}=0.53)$ and $\mathrm{P}(\mathrm{r}=$ $0.59)$. BALP also presented a medium correlation with $\mathrm{Ca}(\mathrm{r}=0.48)$ and $\mathrm{P}(\mathrm{r}=$ $0.60)$. Regarding the correlations obtained over age in the different groups, particularly strong correlations were found in 1 month old lambs, where serum ALP showed correlations with BALP $(\mathrm{r}=0.98 ; \mathrm{P}<0.0001), \mathrm{Ca}(\mathrm{r}=0.85 ; \mathrm{P}<0.001)$ and $\mathrm{P}(\mathrm{r}=0.72 ; \mathrm{P}=0.013)$; BALP was correlated with $\mathrm{Ca}(\mathrm{r}=0.77 ; \mathrm{P}=0.001)$ and $\mathrm{P}(\mathrm{r}=0.92 ; \mathrm{P}=0.008)$, and $\mathrm{Ca}$ was also correlated with $\mathrm{P}(\mathrm{r}=0.57 ; \mathrm{P}=$ $0.01)$. The correlations lost statistical significance over the lifespan of sheep.

\section{Discussion}

Our goal was to perform an extensive study that assesses the variation of serum ALP, BALP and mineral levels, determining reference ranges in sheep of various ages and throughout the different physiological states in ewes, since the measurement of these analytical parameters is frequently cited in the scientific literature when sheep are used in animal science studies and also as a large animal model for orthopaedic research.

In the present study, serum ALP activity was found to be within the reference range (69-387 U/L) (Kaneko et al., 1997) for sheep in all groups, with the exception of mature sheep aged 3 to 5 years or older, and pregnant ewes which presented values slightly below that of the reference range. Serum ALP activity showed the lowest values as sheep got older and, in spite of a higher ALP level in lactating ewes relative to dry or pregnant ones of the same age, no statistically significant differences were found between ewes in different physiologic states.

Concerning serum BALP concentration, the values obtained in this study are similar to those found by Liesegang et al. (2003), who reported a BALP reference value of 35-40 U/L in growing (6 months old) female sheep, those reported by Klein et al. (2004) and Seebeck et al. (2005) in skeletally mature female sheep ( 2 years old), in which the bone healing process was assessed, indicating a BALP reference range of $2.4-33.2 \mu \mathrm{g} / \mathrm{L}$ and $6.4-42.9 \mu \mathrm{g} / \mathrm{L}$ (17-112 U/L), respectively, those reported by Arens et al. (2007) who indicated a serum BALP activity of 25$30 \mathrm{U} / \mathrm{L}$ in healthy female sheep ( 2 to 3 years old) during spring, those found by Dias et al. (2008) who reported $15.0 \pm 5.44$ U/L serum BALP activity in healthy adult sheep (3 years old) under a controlled diurnal photoperiod cycle, and those described by Chavassieux et al. (1997) who indicated a BALP value of $2-4 \mu \mathrm{g} / \mathrm{L}$ in mature ( 8 to 10 years old) ewes used in a preclinical study for osteopenia. The pattern of variation of serum BALP along the sheep lifetime (growing, mature, old, lactating, dry, pregnant) was similar to that described for total ALP, presenting a continuous decline of its serum levels along the sheep lifespan, namely in mature and older sheep ( 3 to 5 years old or older), probably indicating decreased bone 
turnover associated with ageing and metabolism. In the human population and also in dogs, a similar pattern was found not only for BALP but also for other bone formation markers such as OC, PINP and PICP, and also for bone resorption markers (Allen et al., 1998; Allen et al., 2000; Cremers et al., 2008).

In ewes in different physiological states, in spite of the higher level of serum BALP concentration in the pregnant ewes relative to dry or lactating ones of the same age, no statistically significant differences were observed, probably because this is a well-functioning regulatory physiological system. Liesegang et al. (2006) also did not observe significant differences in BALP during gestation and lactation states. These authors found that in milk sheep, after their first gestation, serum BALP activity decreased to a minimum at 4 weeks post partum and then increased again until the 3rd month of lactation, and they reported serum BALP values of around $80 \mathrm{U} / \mathrm{L}$ in mid-pregnancy and around $40 \mathrm{U} / \mathrm{L}$ in the $3 \mathrm{rd}$ month of lactation in young ( 1.5 years old) sheep.

In this study, the sheep showed a wide range of standard deviation for serum ALP activity and BALP concentration over their lifetime (Fig. 1), with particular discrepancy in 1 month old lambs. This fact could be due to the large inter-individual variation for BALP, namely in growing sheep, but also to the liver and intestinal isoenzyme activities which contribute to the total ALP. This aspect has also been observed in previous studies performed in 2 or 3 years old sheep, used as a normal and delayed ovine fracture healing model (Klein et al., 2004; Seebeck et al., 2005). High biological variability is regarded as one of the most important limitations of the application of bone biomarkers (Allen, 2003).

In this study, the values of serum Ca were always slightly below the reference range in all groups, as reported previously by Seebeck et al. (2005) and Dias et al. (2008). The serum $\mathrm{P}$ and $\mathrm{Mg}$ values of all groups were within the normal general reference range published for sheep (Kaneko et al., 1997). Serum Ca showed a slight increase until 2 years of age with a subsequent statistically significant decrease in mature sheep, while serum $\mathrm{P}$ concentration maintained an uninterrupted decrease as sheep aged and $\mathrm{Mg}$ presented an almost constant serum concentration along the sheep lifespan. In 3 to 5 years old ewes of different physiological conditions, serum $\mathrm{Ca}$ was higher in lactating and pregnant ewes than in dry ewes and, on the contrary, $\mathrm{P}$ was lower in lactating and pregnant ewes than in dry ewes, but neither of these differences were statistically significant. Serum Mg was lower in lactating than in dry and pregnant ewes, with a statistically significant difference between lactating and pregnant ewes. These results are again similar to those of Liesegang et al. (2006), who reported serum $\mathrm{Ca}$ concentrations maintained within the reference range in sheep during the first gestation and lactation, in spite of the fact that, during pregnancy and lactation, the flux of Ca resulting from the demands of the fetus or milk production can extract large quantities of this mineral from the blood, requiring adaptive changes in female bone metabolism to maintain Ca homeostasis. 
The significant degree of correlation between serum ALP and BALP levels and between these and $\mathrm{Ca}$ and $\mathrm{P}$ supports the existence of a relationship between these parameters and their involvement in the mineralisation of skeletal tissues. The degree of correlation was dramatically high in 1 month old lambs in which serum ALP and BALP activities were particularly elevated due to high skeletal growth velocity and rapid bone turnover.

Although serum ALP, BALP and mineral levels have been measured in various studies in sheep, to our knowledge no extensive report has been published about the values, pattern of variation and correlations of these parameters throughout the entire sheep lifespan, which are now documented with this study. This knowledge may be useful not only in animal science studies but also in preclinical orthopaedic research using sheep as a large animal model for humans, since the biomarkers of bone metabolism may provide information complementary to the results of imaging, mechanical, histological and histomorphometric analyses.

\section{Acknowledgement}

Cristina P. Sousa acknowledges the Portuguese Foundation for Science and Technology for her PhD scholarship SFRH/BD/45018/2008.

\section{References}

Allen, M. J. (2003): Biochemical markers of bone metabolism in animals: uses and limitations. Vet. Clin. Pathol. 32, 101-113.

Allen, M. J., Allen, L. C. V., Hoffmann, W. E., Richardson, D. C. and Breur, G. J. (2000): Urinary markers of type I collagen degradation in the dog. Res. Vet. Sci. 69, 123-127.

Allen, M. J., Hoffmann, W. E., Richardson, D. C. and Breur, G. J. (1998): Serum markers of bone metabolism in dogs. Am. J. Vet. Res. 59, 250-254.

AOAC (1990): Official Methods of Analysis, 14th edition. Association of Official Analytical Chemists, Gaithersburg, Maryland, USA.

Arens, D., Sigrist, I., Alini, M., Schawalder, P., Schneider, E. and Egermann, M. (2007): Seasonal changes in bone metabolism in sheep. Vet. J. 174, 585-591.

Chavassieux, P., Buffet, A., Vergnaud, P., Garnero, P. and Meunier, P. J. (1997): Short-term effects of corticosteroids on trabecular bone remodeling in old ewes. Bone 20, 451-455.

Cremers, S., Garnero, P. and Seibel, M. J. (2008): Biochemical markers of bone metabolism. In: Bilezikian, J. P., Raisz, L. G. and Martin, T. J. (eds) Principles of Bone Biology. 3rd edition. Academic Press, San Diego, California, USA. pp. 1857-1881.

Dias, I. R., Viegas, C. A., de Azevedo, J. T., Costa, E. M., Lourenco, P., Rodrigues, A. and Cabrita, A. S. (2008): Assessment of markers of bone formation under controlled environmental factors and their correlation with serum minerals in adult sheep as a model for orthopaedic research. Lab. Anim. 42, 465-472.

Ding, M., Cheng, L., Bollen, P., Schwarz, P. and Overgaard, S. (2010): Glucocorticoid induced osteopenia in cancellous bone of sheep: validation of large animal model for spine fusion and biomaterial research. Spine 35, 363-370. 
Egermann, M., Goldhahn, J., Holz, R., Schneider, E. and Lill, C. A. (2008): A sheep model for fracture treatment in osteoporosis: benefits of the model versus animal welfare. Lab. Anim. 42, 453-464.

Goldstein, D. J., Rogers, C. E. and Harris, H. (1980): Expression of alkaline phosphatase loci in mammalian tissues. Proc. Natl Acad. Sci. USA 77, 2857-2860.

Kaneko, J. J., Harvey, J. W. and Bruss, M. L. (1997): Clinical Biochemistry of Domestic Animals. 5th edition. Academic Press, San Diego, California, USA. pp. 890-894.

Klein, P., Bail, H. J., Schell, H., Michel, R., Amthauer, H., Bragulla, H. and Duda, G. N. (2004): Are bone turnover markers capable of predicting callus consolidation during bone healing? Calcif. Tissue Int. 75, 40-49.

Liesegang, A., Risteli, J. and Wanner, M. (2006): The effects of first gestation and lactation on bone metabolism in dairy goats and milk sheep. Bone 38, 794-802.

Liesegang, A., Sassi, M. L. and Risteli, J. (2003): Diurnal variation in concentrations of various markers of bone metabolism in growing female goats and sheep. Anim. Sci. 77, 197-203.

MacLeay, J. M., Olson, J. D. and Turner, A. S. (2004): Effect of dietary-induced metabolic acidosis and ovariectomy on bone mineral density and markers of bone turnover. J. Bone Miner. Metab. 22, 561-568.

Millán, J. L. (2006): Alkaline phosphatases. Purinergic Signal 2, 335-341.

NRC (1985): Nutrient Requirements of Sheep. 6th edition. National Academic Press, Washington D.C., Washington, USA.

Prickett, T. C., Barrell, G. K., Wellby, M., Yandle, T. G., Richards, A. M. and Espiner, E. A. (2008): Effect of sex steroids on plasma C-type natriuretic peptide forms: stimulation by oestradiol in lambs and adult sheep. J. Endocrinol. 199, 481-487.

Seebeck, P., Bail, H. J., Exner, C., Schell, H., Michel, R., Amthauer, H., Bragulla, H. and Duda, G. N. (2005): Do serological tissue turnover markers represent callus formation during fracture healing? Bone 37, 669-677.

Seibel, M. J. (2006): Clinical application of biochemical markers of bone turnover. Arq. Bras. Endocrinol. Metabol. 50, 603-620.

Sigrist, I. M., Gerhardt, C., Alini, M., Schneider, E. and Egermann, M. (2007): The long-term effects of ovariectomy on bone metabolism in sheep. J. Bone Miner. Metab. 25, 28-35.

Turner, A. S. (2007): Seasonal changes in bone metabolism in sheep: Further characterization of an animal model for human osteoporosis. Vet. J. 174, 460-461.

Turner, A. S., Alvis, M., Myers, W., Stevens, M. L. and Lundy, M. W. (1995): Changes in bone mineral density and bone-specific alkaline phosphatase in ovariectomized ewes. Bone 17 (Suppl. 4), S395-S402.

Whyte, M. P. (1994): Hypophosphatasia and the role of alkaline phosphatase in skeletal mineralization. Endocr. Rev. 15, 439-461. 